The Journal of Nonlinear $\mathbf{S}$ ciences and Applications http://www.tjnsa.com

\title{
A CONTRACTION THEOREM IN MENGER PROBABILISTIC METRIC SPACES
}

\author{
S. SHAKERI ${ }^{1}$
}

\begin{abstract}
In this paper, we consider complete menger probabilistic quasimetric space and prove a common fixed point theorem for commuting maps in this space.
\end{abstract}

\section{INTRODUCTION AND PRELIMINARIES}

K. Menger introduced the notion of a probabilistic metric space in 1942 and since then the theory of probabilistic metric spaces has developed in many directions [4]. The idea of K. Menger was to use distribution functions instead of nonnegative real numbers as values of the metric. The notion of a probabilistic metric space corresponds to situations when we do not know exactly the distance between two points, but we know probabilities of possible values of this distance. A probabilistic generalization of metric spaces appears to be interest in the investigation of physical quantities and physiological thresholds. It is also of fundamental importance in probabilistic functional analysis.

In the sequel, we shall adopt usual terminology, notation and conventions of the theory of probabilistic menger metric spaces, as in [4].

Throughout this paper, the space of all probability distribution functions (briefly, d.f.'s) denotes $\Delta^{+}=\{F: \mathbb{R} \cup\{-\infty,+\infty\} \longrightarrow[0,1]: F$ is left-continuous and non-decreasing on $\mathbb{R}, F(0)=0$ and $F(+\infty)=1\}$ and the subset $D^{+} \subseteq \Delta^{+}$is the set $D^{+}=\left\{F \in \Delta^{+}: l^{-} F(+\infty)=1\right\}$. Here $l^{-} f(x)$ denotes the left limit of the function $f$ at the point $x, l^{-} f(x)=\lim _{t \rightarrow x^{-}} f(t)$. The space $\Delta^{+}$is partially ordered by the usual point-wise ordering of functions, i.e., $F \leq G$ if and only if

Date: Received: 2 Aut 2008 ; Revised: 1 Dec 2008.

1 Corresponding author.

2000 Mathematics Subject Classification. 54E70, 54H25.

Key words and phrases. Probabilistic metric spaces; menger space; fixed point theorem; commuting maps; triangle norm. 
$F(t) \leq G(t)$ for all $t$ in $\mathbb{R}$. The maximal element for $\Delta^{+}$in this order is the d.f. given by

$$
\varepsilon_{0}(t)= \begin{cases}0, & \text { if } t \leq 0 \\ 1, & \text { if } t>0\end{cases}
$$

Definition 1.1. A mapping $T:[0,1] \times[0,1] \longrightarrow[0,1]$ is continuous $t$-norm if $T$ is satisfying the following conditions:

(a) $T$ is commutative and associative;

(b) $T$ is continuous;

(c) $T(a, 1)=a$ for all $a \in[0,1]$;

(d) $T(a, b) \leq T(c, d)$ whenever $a \leq c$ and $c \leq d$, and $a, b, c, d \in[0,1]$.

Two typical example of continuous t-norm are $T(a, b)=a b$ and $T(a, b)=$ $\min (a, b)$.

Definition 1.2. A Menger Probabilistic Metric space (briefly, Menger PM-space) is a triple $(X, \mathcal{F}, T)$, where $X$ is a nonempty set, $T$ is a continuous $t$-norm, and $\mathcal{F}$ is a mapping from $X \times X$ into $D^{+}$such that, if $F_{x, y}$ denotes the value of $\mathcal{F}$ at the pair $(x, y)$, the following conditions hold: for all $x, y, z$ in $X$,

(PM1) $F_{x, y}(t)=\varepsilon_{0}(t)$ for all $t>0$ if and only if $x=y$;

(PM2) $F_{x, y}(t)=F_{y, x}(t)$;

(PM3) $F_{x, z}(t+s) \geq T\left(F_{x, y}(t), F_{y, z}(s)\right)$ for all $x, y, z \in X$ and $t, s \geq 0$.

Definition 1.3. Let $(X, \mathcal{F}, T)$ be a PM space.

(1) A sequence $\left\{p_{n}\right\}_{n}$ in $X$ is said to be convergent to $p$ in $X$ if, for every $\epsilon>0$ and $\lambda>0$, there exists positive integer $N$ such that $F_{p_{n}, p}(\epsilon)>1-\lambda$ whenever $n \geq N$.

(2) A sequence $\left\{p_{n}\right\}_{n}$ in $X$ is called Cauchy sequence if, for every $\epsilon>0$ and $\lambda>0$, there exists positive integer $N$ such that $F_{p_{n}, p_{m}}(\epsilon)>1-\lambda$ whenever $n \geq m \geq N(m \geq n \geq N)$.

(3) A PM space $(X, \mathcal{F}, T)$ is said to be complete if and only if every Cauchy sequence in $X$ is convergent to a point in $X$.

Theorem 1.4. [4] If $(X, \mathcal{F}, T)$ is a PM space and $\left\{p_{n}\right\}$ and $\left\{q_{n}\right\}$ are sequences such that $p_{n} \rightarrow p$ and $q_{n} \rightarrow q$, then $\lim _{n \rightarrow \infty} F_{p_{n}, q_{n}}(t)=F_{p, q}(t)$.

Lemma 1.5. Let $(X, \mathcal{F}, \mathcal{T})$ be a $P M$ space. If we define $E_{\lambda, F}: X^{2} \longrightarrow \mathbf{R}^{+} \cup\{0\}$ by

$$
E_{\lambda, F}(x, y)=\inf \left\{t>0: F_{x, y}(t)>1-\lambda\right\}
$$

for each $\lambda \in] 0,1[$ and $x, y \in X$. Then we have

(i) For any $\mu \in] 0,1[$ there exists $\lambda \in] 0,1[$ such that

$$
E_{\mu, F}(x, z) \leq E_{\lambda, F}(x, y)+E_{\lambda, F}(y, z)
$$

for any $x, y, z \in X$;

(ii) The sequence $\left\{x_{n}\right\}_{n \in \mathbb{N}}$ is convergent w.r.t. $\mathcal{F}$ if and only if $E_{\lambda, F}\left(x_{n}, x\right) \rightarrow 0$. Also the sequence $\left\{x_{n}\right\}_{n \in \mathbb{N}}$ is Cauchy w.r.t. $\mathcal{F}$ if and only if it is Cauchy with $E_{\lambda, F}$. 
Proof. For (i), by the continuity of $t$-norms, for every $\mu \in] 0,1[$ we can find a $\lambda \in] 0,1[$ such that

$$
T(1-\lambda, 1-\lambda) \geq 1-\mu
$$

By (PM2) we have

$$
\begin{aligned}
& F_{x, z}\left(E_{\lambda, F}(x, y)+E_{\lambda, F}(y, z)+2 \delta\right) \\
\geq & T\left(F_{x, y}\left(E_{\lambda, F}(x, y)+\delta\right), F_{y, z}\left(E_{\lambda, F}(y, z)+\delta\right)\right) \\
\geq & T(1-\lambda, 1-\lambda) \\
\geq & 1-\mu
\end{aligned}
$$

for every $\delta>0$, which implies that

$$
E_{\mu, F}(x, z) \leq E_{\lambda, F}(x, y)+E_{\lambda, F}(y, z)+2 \delta .
$$

Since $\delta>0$ was arbitrary, we have

$$
E_{\mu, F}(x, z) \leq E_{\lambda, F}(x, y)+E_{\lambda, F}(y, z) .
$$

For (ii), we have

$$
F_{x_{n}, x}(\eta)>1-\lambda \Longleftrightarrow E_{\lambda, F}\left(x_{n}, x\right)<\eta
$$

for every $\eta>0$.

\section{A COMmon FIXED POINT THEOREM FOR COMMUTING MAPS}

Theorem 2.1. Let $f$ be a continuous mapping of a complete metric space $(X, d)$ into itself and let $g: X \longrightarrow X$ be a map that satisfy the following conditions:

(a) $g(X) \subseteq f(x)$;

(b) $g$ commutes with $f$;

(c) $d(g(x), g(y)) \leq k d(f(x), f(y))$ for all $x, y \in X$ and for some $0<k<1$.

Then $f$ and $g$ have a unique common fixed point.

The above result has a probabilistic analogue in the following theorem.

Theorem 2.2. Let $(X, \mathcal{F}, T)$ be a complete PM space and let $f, g: X \longrightarrow X$ be maps that satisfy the following conditions:

(a) $g(X) \subseteq f(x)$;

(b) $f$ is continuous;

(c) $F_{g(x), g(y)}(k t) \geq F_{f(x), f(y)}(t)$ for all $x, y \in X$ and $0<k<1$.

Then $f$ and $g$ have a unique common fixed point provided $f$ and $g$ commute.

Proof. Let $x_{0} \in X$. By (a) we can find $x_{1}$ such that $f\left(x_{1}\right)=g\left(x_{0}\right)$. By induction, we can define a sequence $\left\{x_{n}\right\}_{n}$ such that $f\left(x_{n}\right)=g\left(x_{n-1}\right)$. By induction again,

$$
\begin{aligned}
F_{f\left(x_{n}\right), f\left(x_{n+1}\right)}(t) & =F_{g\left(x_{n-1}\right), g\left(x_{n}\right)}(t) \geq F_{f\left(x_{n-1}\right), f\left(x_{n}\right)}(t / k) \\
& \geq \cdots \geq F_{f\left(x_{0}\right), f\left(x_{1}\right)}\left(t / k^{n}\right)
\end{aligned}
$$


for $n=1,2, \cdots$, which implies that

$$
\begin{aligned}
E_{\lambda, F}\left(f\left(x_{n}\right), f\left(x_{n+1}\right)\right) & =\inf \left\{t>0: F_{f\left(x_{n}\right), f\left(x_{n+1}\right)}(t)>1-\lambda\right\} \\
& \leq \inf \left\{t>0: F_{f\left(x_{0}\right), f\left(x_{1}\right)}\left(t / k^{n}\right)>1-\lambda\right\} \\
& =k^{n} \inf \left\{t>0: F_{f\left(x_{0}\right), f\left(x_{1}\right)}(t)>1-\lambda\right\} \\
& =k^{n} E_{\lambda, F}\left(f\left(x_{0}\right), f\left(x_{1}\right)\right)
\end{aligned}
$$

for every $\lambda \in] 0,1[$.

Now, we show that $\left\{f\left(x_{n}\right)\right\}$ is a Cauchy sequence. For every $\left.\mu \in\right] 0,1[$, there exists $\gamma \in] 0,1[$ such that, for $m \geq n$,

$$
\begin{aligned}
& E_{\mu, F}\left(f\left(x_{n}\right), f\left(x_{m}\right)\right) \\
\leq & E_{\gamma, F}\left(f\left(x_{m-1}, f\left(x_{m}\right)\right)+E_{\gamma, F}\left(f\left(x_{m-2}\right), f\left(x_{m-1}\right)\right)+\cdots+E_{\gamma, F}\left(f\left(x_{n}\right), f\left(x_{n+1}\right)\right)\right. \\
\leq & E_{\gamma, F}\left(f\left(x_{0}, f\left(x_{1}\right)\right) \sum_{j=n}^{m-1} k^{J} \longrightarrow 0\right.
\end{aligned}
$$

as $m, n \longrightarrow \infty$. Since $X$ is left complete, there exists $y \in X$ such that $\lim _{n \rightarrow \infty} f\left(x_{n}\right)=$ $y$. So $g\left(x_{n-1}\right)=f\left(x_{n}\right)$ tends to $y$. It can be seen from (c) that the continuity of $f$ implies that to $g$.

So $\left\{g\left(f\left(x_{n}\right)\right)\right\}_{n}$ converges to $g(y)$. However, $g\left(f\left(x_{n}\right)\right)=f\left(g\left(x_{n}\right)\right)$ by the commutativity of $f$ and $g$. So $f\left(g\left(x_{n}\right)\right)$ converges to $f(y)$. Because the limits are unique, $f(y)=g(y)$. So $f(f(y))=f(g(y))$ by commutativity and

$$
\begin{aligned}
F_{g(y), g(g(y))}(t) & \geq F_{f(y), f(g(y))}(t / k) \geq F_{g(y), g(g(y))}(t / k) \\
& \geq \cdots \geq F_{g(y), g(g(y))}\left(t / k^{n}\right) \longrightarrow 1
\end{aligned}
$$

as $n \rightarrow \infty$, which implies that, $g(y)=g(g(y))$. Thus, $g(y)=g(g(y))=f(g(y))$. So $g(y)$ is a common fixed point of $f$ and $g$.

If $y$ and $z$ are two fixed points common to $f$ and $g$, then

$$
\begin{aligned}
\varepsilon_{0}(t) & \geq F_{y, z}(t)=F_{g(y), g(z)}(t) \geq F_{f(y), f(z)}(t / k) \\
& =F_{y, z}(t / k) \cdots \geq F_{y, z}\left(t / k^{n}\right) \longrightarrow 1 .
\end{aligned}
$$

as $n \rightarrow \infty$, which implies that, $y=z$.

Example 2.3. Let $X$ be the set $\left\{\frac{1}{n}: n \in \mathbb{N}\right\} \cup\{0\}$. Denote $T(a, b)=a b$ for all $a, b \in[0,1]$. For each $t \in] 0, \infty[$, define

$$
F_{x, y}(t)=\left\{\begin{array}{cl}
\frac{t}{t+|x-y|}, & \text { if } t>0, \\
0, & \text { if } t=0 .
\end{array}\right.
$$

Clearly $(X, \mathcal{F}, T)$ is a complete PM space. Define $g(x)=x / 12$ and $f(x)=x / 4$ on $X$. It is evident that $g(X) \subseteq f(X)$. Also for $k=1 / 3$,

$$
F_{g(x), g(y)}(t / 3)=\frac{t / 3}{t / 3+(|x-y| / 12)}=\frac{t}{t+|x-y| / 4}=F_{f(x), f(y)}(t) .
$$

Thus all the conditions of Theorem 2.2 are satisfied and $f$ and $g$ have the common fixed point 0 . 


\section{REFERENCES}

1. S. S. Chang,Y. J. Cho and S. M. Kang, Nonlinear Operator Theory in Probabilistic Metric Spaces, Nova Science Publishers, Inc., New York, 2001.

2. O. Hadžić, E. Pap, Fixed Point Theory in PM Spaces, Kluwer Academic Publishers, Dordrecht, 2001.

3. D. Mihet, V. Radu, On the stability of the additive Cauchy functional equation in random normed spaces, J. Math. Anal. Appl. 343 (2008) 567-572.

4. B. Schweizer and A. Sklar, Probabilistic Metric Spaces, Elsevier, North Holand, New York, 1983.

1, 1.4

Department of Mathematics

Islamic Azad University-Ayatollah Amoly Branch

Amol P. O. Box 678, Amol, Iran. 